\title{
La administración de selenio disminuye la lipoperoxidación en semen de toros Brahman
}

\author{
Flores, C.; Márquez, Y.; Vilanova, L.; Matheus, N.; López Ortega, A. \\ Unidad de Investigación en Ciencias Funcionales Dr. Haity Moussatché (UNIHM), Facultad de Ciencias \\ Veterinarias, Universidad Centroccidental "Lisandro Alvarado" (UCLA), Barquisimeto (Lara, Venezuela). \\ E-mail: caf06@hotmail.com
}

\begin{abstract}
Resumen
Flores, C.; Márquez, Y.; Vilanova, L.; Matheus, N.; López Ortega, A.: La administración de selenio disminuye la lipoperoxidación en semen de toros Brahman. Rev. vet. 25: 2, 95-99, 2014. La lipoperoxidación es uno de los efectos del estrés oxidativo, el cual cursa con alteraciones de motilidad y viabilidad espermática debido a cambios bioquímicos y estructurales de la membrana plasmática. El trastorno conduce a la infertilidad, por lo cual es menester estudiar las alternativas terapéuticas para mejorar las condiciones reproductivas de los toros. En este estudio se determinó la acción antioxidante del selenio y sus efectos sobre la calidad seminal. Se emplearon toros Brahman sanos, de 15-18 meses de edad, divididos en dos grupos mantenidos a pastoreo. El grupo experimental recibió una dosis intramuscular de $0,22 \mathrm{mg} / 20 \mathrm{~kg} \mathrm{PV} /$ día durante 5 días. Las muestras fueron tomadas con electroeyaculador a los días cero, 15 y 30 post-tratamiento. Se evaluó la calidad seminal (espermatozoides móviles, porcentaje de motilidad progresiva, morfología, vitalidad y presencia de acrosomas) e indicadores de la peroxidación lipídica: dienos conjugados (DC, extraídos con isopropanol) y malondialdehído (MDA, por TBARS). Los datos fueron interpretados a través del análisis de la variancia $(\mathrm{p} \leq 0,05)$. La calidad del semen no reveló diferencias significativas entre grupos. Los animales tratados no mostraron diferencias significativas en los DC a los 15 y 30 días con respecto al grupo control. Por su parte, el MDA presentó diferencias significativas a los 30 días de tratamiento, cuando el grupo experimental mostró valores más bajos $(1,09 \pm 0,1 \mathrm{nmoles} / \mathrm{mg}$ de proteínas) con respecto al grupo control (1,58 $\pm 0,2 \mathrm{nmoles} / \mathrm{mg}$ de proteínas). Se concluye que el selenio resulta eficaz para reducir la lipoperoxidación seminal y preservar la calidad del semen.
\end{abstract}

Palabras clave: toro Brahman, lipoperoxidación, infertilidad, selenio, calidad seminal.

\begin{abstract}
Flores, C.; Márquez, Y.; Vilanova, L.; Matheus, N.; López-Ortega, A. Administration of selenium decreases lipid peroxidation in semen of Brahman bulls. Rev. vet. 25: 2, 95-99, 2014. Lipid peroxidation, one of the effects of oxidative stress, is associated with impaired sperm motility and viability because of biochemical and structural alterations of the plasma membrane which causes infertility. Of interest is the study of therapeutic alternatives for enhancing the reproductive condition of the bulls. In this study the antioxidant action of selenium and its effect on semen quality was determined. Healthy grazing Brahman bulls with 15-18 months of age, divided into two groups, were used for the trial. The experimental group received an intramuscular dose of $0.22 \mathrm{mg} / 20 \mathrm{~kg}$ liveweight/day, during 5 days. The sample was taken with electroejaculator at day zero, 15 and 30 post-treatment. Diene conjugates (DC, extracted with isopropanol) and malondialdehyde (MDA TBARS) were measured. Semen quality (motile sperm, percentage of progressive motility, morphology, sperm vitality and presence of acrosomes) and indicators of lipid peroxidation were evaluated. Data were analyzed by ANOVA $(\mathrm{p} \leq 0.05)$. In the study of semen, quality groups did not differ significantly. Compared to the control group, selenium-treated animals showed no significant differences in DC at 15 and 30 days. On the other hand, MDA revealed significant differences at 30 days post treatment, showing lower values $(1.09 \pm 0.1 \mathrm{nmol} / \mathrm{mg}$ protein) compared to control group (1.58 $\pm 0.2 \mathrm{nmoles} / \mathrm{mg}$ protein). In conclusion, seminal selenium decreases lipid peroxidation and preserves semen quality.
\end{abstract}

Key words: Brahman bull, lipid peroxidation, infertility, selenium, seminal quality. 


\section{INTRODUCCIÓN}

La calidad seminal se establece al comparar pruebas de laboratorio con parámetros considerados normales para un toro reproductor adulto. Tales "valores normales" están directamente relacionados con la fertilidad y permiten seleccionar los machos superiores, no sólo por buena libido sino también por poseer un semen de elevada calidad y fertilidad ${ }^{25}$. La evaluación toma en cuenta el nivel de motilidad y morfología espermática, pruebas que además indican la habilidad de los espermatozoides para sobrellevar los procesos de congelado y descongelado ${ }^{2}$.

La evaluación seminal en toros seleccionados como reproductores es fundamental para evitar problemas de sub-fertilidad e infertilidad en el rebaño ${ }^{25} \mathrm{y}$ en el caso de los futuros sementales siempre es conveniente la caracterización de las variables espermáticas en cada uno de los meses de edad a partir del arribo a la pubertad, pues ello permitirá establecer los indicadores de normalidad, hacer un pronóstico de los toros que no tienen aptitudes como reproductores e incluir estos indicadores dentro de los parámetros para la selección ${ }^{21}$.

La acción de los radicales libres (RL) en el semen, como la lipoperoxidacion de la membrana plasmática, produce cambios en la fluidez de la misma y altera profundamente el comportamiento espermático generando problemas de migración, capacitación, unión y fusión de los gametos ${ }^{3}$. Los espermatozoides son más susceptibles al daño peroxidativo de los RL (cuyo exceso define el estado de estrés oxidativo) debido a sus altas concentraciones de ácidos grasos poliinsaturados en la membrana espermática ${ }^{22}$. Además, los espermatozoides poseen escasa cantidad de citoplasma, lo cual limita la acción antioxidante de los sistemas enzimáticos ${ }^{3}$ que no logran atenuar eficientemente la producción excesiva de $\mathrm{RL}^{15}$.

Las enzimas antioxidantes se caracterizan por requerir cofactores como manganeso, zinc, magnesio, cobre o selenio para su buen funcionamiento ${ }^{14}$. Tal es el caso de la metaloenzima glutatión peroxidasa, que contiene 4 subunidades, cada una de ellas unidas a selenio, el cual es esencial para su actividad. Las deficiencias de este mineral pueden inducir modificaciones del estado oxidativo celular y a la aparición de enfermedades ${ }^{4,8}$, en el caso del bovino la enfermedad del músculo blanco y la retención placentaria, entre otras 5,23 .

En Venezuela está limitado el contenido de selenio en la dieta de los bovinos, debido a que los pastos constituyen (casi exclusivamente) su única fuente de nutrientes. Además, suelos y pasturas presentan desequilibrios en la cantidad de minerales, que causan efectos negativos en los procesos reproductivos de los rumiantes, circunstancia que se refleja en el incremento de la mortalidad y disminución de la natalidad ${ }^{17}$ y se traduce en pérdidas económicas para los productores.

Por todo lo expuesto, resulta relevante el estudio de alternativas terapéuticas para mejorar las condiciones reproductivas de los toros de alta calidad genética. Esta investigación se orientó a determinar si la suplementación con selenio poseía acción antioxidante, evaluando sus efectos sobre la calidad seminal en toros Brahman jóvenes.

\section{MATERIAL Y MÉTODOS}

Area de estudio. El trabajo fue realizado en el Centro Genético de Raza Brahman ubicado en las coordenadas $09^{\circ} 40^{\prime} 45^{\prime \prime}$ y $10^{\circ} 02^{\prime} 35^{\prime}$ " de latitud norte y los $68^{\circ}$ $48^{\prime} 14^{\prime \prime}$ y $69^{\circ} 16^{\prime} 52^{\prime \prime}$ de longitud oeste, en la carretera Barquisimeto-Acarigua Km. 30, vía Gamelotal, caserío Quebrada Honda, Parroquia Sarare, Municipio Simón Planas en el Estado de Lara (Venezuela), zona caracterizada por un ecosistema de bosque seco tropical ${ }^{7,16}$. La vegetación incluye herbáceas en donde la temperatura promedio de la zona es de aproximadamente $26,2^{\circ} \mathrm{C}$ a $285 \mathrm{msnm}$, el promedio anual de precipitación es de $1421,3 \mathrm{~mm}$, con registro de máxima lluviosidad durante los meses de mayo a octubre y la mínima durante los meses de diciembre a marzo.

Población y muestra. A partir de un plantel de 235 bovinos Brahman puros (Bos indicus), fueron seleccionados al azar, 31 toros de edades comprendidas entre los 15 y 18 meses. Luego de tres estudios andrológicos se eliminaron 6 animales por presentar azoospermia. De los 25 toretes restantes, 13 se utilizaron como grupo experimental y 12 como controles. Las evaluaciones andrológicas se realizaron cada 15 días hasta completar dos evaluaciones, obteniéndose el semen mediante el uso de electroeyaculador.

Tratamiento. El tratamiento consistió en la administración de una solución comercial de selenio a una concentración del $0,2 \%$, recibiendo cada animal del grupo experimental $0,22 \mathrm{mg}$ por cada $20 \mathrm{~kg}$ de peso corporal vía intramuscular diariamente durante 5 días, dosis recomendada por el fabricante del producto. Los controles fueron inyectados con solución salina.

Circunferencia escrotal. Se tomó la medida testicular con una cinta métrica especial para estos fines. Las mediciones fueron efectuadas poco antes de realizar la extracción del semen con electroeyaculador.

Calidad seminal. Los parámetros evaluados fueron: motilidad espermática progresiva, porcentaje de espermatozoides móviles, morfología espermática e integridad acrosómica (coloración de Karras) y vitalidad espermática (eosina-nigrosina). Todas las pruebas se efectuaron al momento de la extracción y con microscopia de luz. Las proteínas totales se determinaron mediante el kit Bio Rad (Richmond, Ca, EEUU) basado en el método de Bradford ${ }^{1}$.

Determinación de indicadores de lipoperoxidacion. Para cuantificar DC y MDA, las muestras de semen se conservaron con leche descremada al 1\% (Pas- 
Tabla 1. Calidad seminal y circunferencia escrotal a los 15 y 30 días post-tratamiento.

\begin{tabular}{|c|c|c|c|c|c|c|}
\hline \multirow{2}{*}{ parámetro evaluado } & \multicolumn{3}{|c|}{ control $(n=12)$} & \multicolumn{3}{|c|}{ experimental $(n=13)$} \\
\hline & 0 días & 15 días & 30 días & 0 días & 15 días & 30 días \\
\hline espermatozoides vivos (\%) & $* 22 \pm 3$ & $25 \pm 7$ & $34 \pm 3$ & $* 23 \pm 3$ & $27 \pm 4$ & $34 \pm 5$ \\
\hline motilidad progresiva (\%) & $* 19 \pm 4$ & $22 \pm 6$ & $26 \pm 2$ & $* 19 \pm 2$ & $20 \pm 4$ & $26 \pm 2$ \\
\hline vitalidad espermática (\%) & $* 36 \pm 5$ & $38 \pm 6$ & $55 \pm 4$ & $* 35 \pm 4$ & $40 \pm 4$ & $54 \pm 2$ \\
\hline anomalías espermáticas (\%) & $* 63 \pm 3$ & $60 \pm 4$ & $50 \pm 3$ & $* 65 \pm 2$ & $60 \pm 2$ & $52 \pm 4$ \\
\hline circunferencia escrotal $(\mathrm{cm})$ & $24,5 \pm 0,4$ & $27,8 \pm 0,3$ & $28,3 \pm 0,4$ & $26,5 \pm 0,4$ & $29,3 \pm 0,2$ & $29,8 \pm 0,3$ \\
\hline
\end{tabular}

Valores en media aritmética \pm desvío estándar. *Indica diferencias significativas $(\mathrm{p} \leq 0,05)$ entre valores del día 0 con respecto a los 15 y 30 días, dentro de cada grupo.

toreña ${ }^{\circledR}$ UHT) en una proporción de 1:1 y congeladas a $-20^{\circ} \mathrm{C}$ hasta su procesamiento. En el caso de los DC se realizó la extracción con $900 \mu 1$ de isopropanol, para $100 \mu 1$ de muestra diluida (1:40), en un volumen final de $1 \mathrm{ml}$, mezclado en vortex durante dos minutos y centrifugado a $3500 \mathrm{G}$ durante 5 minutos a $15^{\circ} \mathrm{C}$. La absorbancia del sobrenadante (capa orgánica) fue leída a $232 \mathrm{~nm}$. Para los cálculos, se utilizó el coeficiente de extinción molar $27000 \mathrm{M}^{-1} / \mathrm{L}$, expresando las concentraciones en moles de DC por mg de proteínas.

Por su parte, el MDA se cuantificó mediante el test para sustancias reaccionantes con el ácido 2-tio-barbitúrico (TBARS). Para ello se utilizaron $100 \mu \mathrm{l}$ de muestra y se agregaron de manera sucesiva $1,5 \mathrm{ml}$ de ácido acético glacial al $20 \%(\mathrm{pH} 3,5), 200 \mu \mathrm{l}$ de dodecil sulfato de sodio al $8,1 \%$ y $1,5 \mathrm{ml}$ de ácido tiobarbitúrico al 0,8\%. Los tubos con tapa sin enroscar se incubaron a $95^{\circ} \mathrm{C}$ durante 60 minutos y se enfriaron por 8 minutos en agua a $18^{\circ} \mathrm{C}$, posteriormente se agregó $1 \mathrm{ml}$ de agua destilada y $5 \mathrm{ml}$ de butanol, para luego ser centrifugados a 3000 $\mathrm{G}$ durante 10 minutos, el sobrenadante fue leído con espectrofotómetro Genesys 5 a $532 \mathrm{~nm}$. Para realizar los cálculos se utilizó una curva estándar con 1,1,3,3-tetrametoxipropano como patrón. La concentración de MDA se expresó en nmoles de malondialdehído por mg de proteínas.

Análisis estadístico. Los datos de calidad seminal fueron presentados en porcentaje, mientras que los de DC y MDA se determinaron en mmoles de DC por $\mathrm{mg}$ de proteínas y nmoles de MDA por mg de proteínas. Los datos se analizaron mediante ANOVA, con paquete estadístico SPSS versión 15.0 para Windows, con una $\mathrm{p} \leq 0,05$.

\section{RESULTADOS}

La Tabla 1 indica que al comparar el grupo control con el experimental a los 0 ; 15 y 30 días post-tratamiento con $\mathrm{Se}$, no hubo diferencias significativas en cuanto a calidad seminal. Sin embargo, al realizar la comparación dentro de cada grupo, se evidenciaron cambios significativos en todos los parámetros, con un aumento en los porcentajes de espermatozoides vivos, motilidad progresiva y vitalidad espermática, así como disminución en las anomalías espermáticas, tanto en el grupo control como en el experimental. Por su parte, la circunferencia escrotal mostró una tendencia al aumento lineal durante los 30 días de experimentación, tanto en el grupo control como en el inyectado con Se, sin diferencias significativas entre ambos.

La Figura 1 muestra que los niveles seminales de DC no presentaron diferencias significativas entre los grupos control y experimental a los $0 ; 15$ y 30 días posttratamiento, sin embargo se pudo observar que el grupo

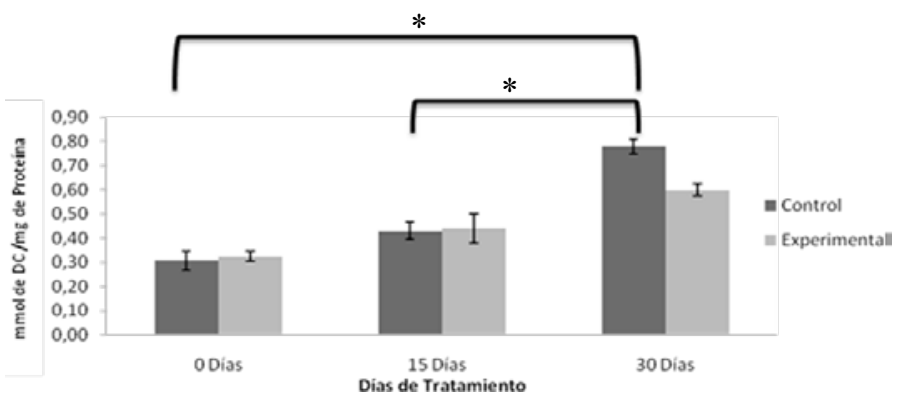

Figura 1. Niveles de dienos conjugados en semen a los 15 y 30 días post-tratamiento (media aritmética \pm desvío estándar). *Indica diferencias significativas $(\mathrm{p} \leq 0,05)$ del grupo control a los 30 días con respecto del grupo control a los 0 y 15 días y experimental antes del tratamiento.

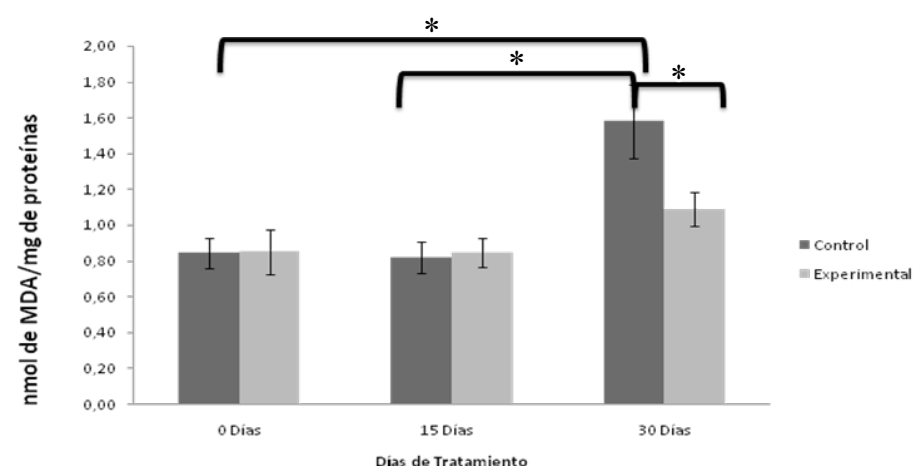

Figura 2. Niveles de malondialdehido en semen a los 15 y 30 días post-tratamiento (media aritmética \pm desvío estándar). *Indica diferencias significativas $(\mathrm{p} \leq 0,05)$ del grupo control a los 30 días con respecto al grupo experimental a los 30 días, así como con el grupo control a los 0 y 15 días. 
control exhibió un aumento significativo en sus niveles a los 30 días.

La Figura 2 revela que los niveles seminales de MDA disminuyeron de manera significativa en el grupo experimental a los a los 30 días post-tratamiento. Además, al realizar comparaciones dentro de cada grupo, en los controles se pudieron evidenciar aumentos significativos de las concentraciones de MDA después de 30 días, no así en el grupo experimental.

\section{DISCUSIÓN}

En esta investigación la administración de selenio por vía intramuscular a la dosis referida ut supra, no modificó la calidad seminal en toros Brahman. No obstante, al realizar la comparación intragrupal se pudieron verificar cambios significativos, con un aumento lineal en la circunferencia escrotal durante el periodo de estudio, lo cual pudiera estar relacionado con cambios fisiológicos relacionados con la edad, tal como lo proponen otros autores, cuyos estudios revelan una correlación positiva, entre el tamaño testicular y la producción seminal en toros menores de tres años de edad ${ }^{6}$. Además, los parámetros seminales se encontraban dentro de los valores normales de acuerdo a la edad, según reportes de investigaciones anteriores ${ }^{11,21}$.

Por otro lado, la suplementación de este mineral no generó cambios en la calidad y cantidad de los eyaculados frescos de cerdos, ni tampoco en el semen almacenado tras su recogida ${ }^{13}$. Sin embargo, se ha publicado que la combinación de selenio con omega 3-6 y probióticos o vitamina $\mathrm{E}$, produjeron en verracos reproductores una disminución en el porcentaje de anormalidades espermáticas ${ }^{24}$. En gallos se constató que la administración de un suplemento de selenio orgánico tuvo efectos significativos sobre la movilidad y la viabilidad de los espermatozoides ${ }^{9}$. En seres humanos infértiles, la administración de selenio combinado con vitamina $\mathrm{E}$, logró aumentar la motilidad espermática ${ }^{12}$.

En cuanto a los indicadores de estrés oxidativo seminal, cabe señalar que los DC se originan por reordenamiento de los dobles enlaces de los peróxidos formados por la acción de radicales libres al comienzo de la oxidación de los ácidos grasos insaturados, constituyentes de estructuras celulares. A medida que la reacción de oxidación avanza, la capacidad de las muestras de absorber los rayos UV disminuye, por el paso de los DC a productos terminales de la lipoperoxidación (entre éstos el MDA), lo que causará lecturas menores a la hora de cuantificar las muestras ${ }^{18}$.

$\mathrm{Al}$ analizar los resultados obtenidos en la presente investigación, se advierte que ambos grupos (control y experimental) presentaron concentraciones de DC sin diferencias significativas antes de iniciar la experiencia (tiempo 0), hallazgo que se mantuvo a los 15 días postadministración intramuscular de Se, pero al transcurrir los 30 días de post-tratamiento, el grupo control mostró un repunte de los niveles de DC seminales, significativamente mayores $(p<0,05)$ al comparar con los valores del grupo control a los 0 y 15 días post-suplementación de Selenio (Figura 1).

De igual manera, en este estudio se encontró que los niveles de MDA seminal presentaron una evolución semejante a lo descrito para DC hasta los 15 días postadministración de Se, para modificarse notablemente a los 30 días post-tratamiento, con una significativa disminución $(\mathrm{p}<0,05)$ de la concentración de MDA seminal en el grupo inyectado con Se. El nivel de MDA en el grupo control a los 30 días post-suplementación fue mayor $(p<0,05)$ al observado en los controles a los 0 y 15 días, tal como es mostrado en la Figura 2.

Resultados similares fueron obtenidos en estudios realizados en seres humanos y en gallos, en los cuales la suplementación de selenio combinado con vitamina E fue capaz de reducir la concentración de MDA ${ }^{12,20}$. El mismo efecto se obtuvo en gansos, particularmente en la segunda mitad de la temporada reproductiva ${ }^{10}$. A pesar de que los niveles séricos de selenio no correlacionaron con la concentración espermática del semen de toros, la administración de adecuados niveles de selenio resultaron fundamentales para mantener bajos índices de lipoperoxidación, tanto en semen fresco como conservado, debido a una mayor actividad de la glutatión peroxidasa ${ }^{19}$.

En base a los resultados, se puede concluir que el selenio administrado por vía intramuscular cada 24 horas durante 5 días, disminuye la lipoperoxidación seminal preservando la calidad seminal, fenómeno puesto de relieve por la disminución de las concentraciones de malondialdehído. El hallazgo implica la posibilidad de utilizar terapéuticamente dicho oligoelemento en los toros afectados por dicha patología.

Agradecimientos. Nuestra gratitud a los profesores Flordaines Colmenares (Facultad de Ciencias Veterinarias, Universidad del Zulia, Venezuela) y Carmen. Meléndez (Unidad de Investigación en Ciencias Funcionales Dr. Haity Moussatché, Barquisimeto) quienes por su importante contribución intelectual y material deben ser considerados coautores de este trabajo, no pudiendo figurar como tales por limitaciones reglamentarias de la revista. El reconocimiento se hace extensivo al profesor Rafael Bonfante-Cabarcas del Doctorado de Ciencias Biomédicas de la Facultad de Ciencias de la Salud de la UCLA y al Consejo de Desarrollo Científico, Humanístico y Tecnológico (CDCHT) y de la Dirección de Investigación de la Universidad Centroccidental Lisandro Alvarado por el financiamiento del proyecto.

\section{REFERENCIAS}

1. Bradford MA. 1976. A rapid and sensitive method for the quantities of microgram of protein utilizing the principle of protein-dye binding. Anal Biochem 72: 248-254.

2. Brogliatti GM. 2013. El impacto de calidad seminal en programas de IAIF. Informe NCPBA.http://www.abspecplan.com.br/upload/library/impacto_seminal.pdf 
3. Bouvet BR, Paparella CV, Feldman RN. 2007. Estres oxidativo y su efecto sobre la calidad seminal. Revista $\mathrm{Ha}$ banera de Ciencias Médicas 6: 1-8.

4. Céspedes CY, Sánchez D. 2000. Algunos aspectos sobre el estrés oxidativo, el estado antioxidante y la terapia de suplementación. Rev Cubana Cardiol 14: 55-60.

5. Eicken K, Scholz H, Stockhofe N. 1992. Mangelhafte selen und vitamin-E versorgung als ursache für bestandsweise auftretende peritarsitiden beim Rind, Tierärztl Umschau 47: 843-847.

6. Espitia A, Prieto E, Cardozo J. 2006. Pubertad y circunferencia escrotal en toros Holstein x Cebú, Cebú y Romosinuano. Revista MVZ (España) 11: 744-750.

7. Ew JJ, Arnold M, Tosi JP. 1976. Zonas de vida de Venezuela. En: Memoria explicativa sobre el mapa ecológico, $2^{\circ}$ ed., Ed. Sucre, Caracas, p. 76-88.

8. Hernández H, Rios MJ. 2009. Rol biológico del selenio en el humano. Química Viva 2: 64-79.

9. Jafari Y, Parizadian B, Zamani M. 2013. The impact of organic selenium supplementation on rooster semen quality in liquid condition. Poultry Sci J 1: 21-28.

10. Jerysz A, Lukaszewicz E. 2013. Effect of dietary selenium and vitamin E on ganders' response to semen collection and ejaculate characteristics. Biol Trace Elem Res 153: 196-204.

11. Jiménez H, González T, Menéndez M. 2002. Efecto de la inmunización contra testosterona sobre las características testiculares del Cebú. Téc Pec Méx 40: 93-104.

12. Keskes L, Feki N, Rebai T, Sahnoun Z, Ghozzi H, Hammami S, Zghal K, Fki H, Damak J, Bahloul A. 2003. Sperm oxidative stress and the effect of an oral vitamin $\mathrm{E}$ and selenium supplement on semen quality in infertile men. Arch Androl 49: 83-94.

13. Lovercamp K, Stewart K, Lin X, Flowers W. 2013. Efecto del selenio de la dieta sobre la calidad de los espermatozoides porcinos. Anim Reprod Sci http://www.3tres3. com/abstracts/efecto-del-selenio-de-la-dieta-sobre-la-calidad-de-los-espermatozoides_32235/

14. Lozada SM, García L. 2009. Estrés oxidativo y antioxidantes: cómo mantener el equilibrio Rev Asoc Colomb Dermatol 17: 172-179.
15. Palamanda JR, Kehrer JP. 1992. Inhibition of protein carbonyl formation and lipid peroxidation by glutathione in rat liver microsomes. Arch Biochem Biophys 293: 103109.

16. PROINLARA. Atlas de Lara, Venezuela, edición 2012. www.proinlara.com

17. Salamanca A. 2010. Suplementación de minerales en la producción bovina. Red Vet 11: 9.

18. Sánchez G. 2012. Caracterización y cuantificación de los ácidos grasos omega 3 y omega 6 presentes en el aceite de sacha inchi (Plukenetia volubilis). Tesis MSci Univ Nac Colombia, http://www.bdigital.unal.edu. co/9141/1/3496371.2012.pdf

19. Slaweta R, Wasowicz W, Laskowska T. 2010. Selenium content, glutathione peroxidase activity, and lipid peroxide level in fresh bull semen and its relationship to motility of spermatozoa after freezing-thawing. J Vet Med Series A 35: 1-10.

20. Surai P, Kostjuk I, Wishart G, MacPherson A, Speake B, Noble R, Ionov I, Kutz E. 1998. Effect of vitamin E and selenium supplementation of cockerel diets on glutathione peroxidase activity and lipid peroxidation susceptibility in sperm, testes, and liver. Biol Trace Elem Res 64: 119-132.

21. Tamayo M. 2013.La selección de sementales bovinos en Cuba. Calidad de la producción seminal en futuros sementales Holstein. Red Vet 14: 1.

22. Tortolero I, Arata G, Osuna JA, Roald, Regadera J. 2005. Estrés oxidativo y función espermática. Rev Venez Endocrinol Metab 3: 12-19.

23. Van Saun RJ. 1990. Rational approach to selenium supplementation essential, Feedstuffs 15: 15-17.

24. Velástegui MP, Tinillo DP. 2011. Evaluación espermática en verracos reproductores mediante la utilización de suplementos: ácidos omega 3-6 con selenio orgánico y probióticos con vitamina E. Tesis Med Vet, Univ Cotopaxi, Unidad Cs Agropec., Ecuador. http://repositorio.utc.edu. ec/bitstream/27000/688/1/T-UTC-0548.pdf

25. Vera O. 2001. Evaluación seminal comparativa pre y postcongelación en machos bovinos. En: Reproducción Bovina (Fund. Giraz Ed.), Maracaibo, p. 251-262 . 